\title{
Mobile Age: Co-creating Digital Public Services with and for Older Citizens
}

\section{Introduction to the Project}

The co-creation projects reported in this book were part of the EU-funded project Mobile Age $^{1}$ in which digital public services were co-created with substantial participation of older citizens. The overall aim of Mobile Age was to develop and test methods for co-creating open digital services for age-friendly cities and communities. This included objectives such as

- enabling civic open data use of older adults,

- increasing digital inclusion of older adults, and

- co-creating sustainable digital public services for older adults.

So far, older adults are using the internet and in particular, eGovernment services to a much lesser degree than other age groups (digital divide as age divide). Mobile Age assumed that a way to make digital services more attractive and beneficial for older adults was to involve them in the process of identifying, conceptualising and designing relevant and usable digital services. As such, Mobile Age followed and extended an approach to co-creation that exceeds traditional ways of citizen participation. It explored, developed and tested new methods and tools.

This book is based on three of the six co-creation projects conducted in Mobile Age: two in Bremen, Germany and one Zaragoza, Spain. In a first phase, a pilot cocreation project was conducted in Bremen (district Osterholz) from May 2016 to February 2017. The learnings from this project fed into the planning and implementation of two further projects from May 2017 to February 2018: In a second district in Bremen (Hemelingen) and in Zaragoza to experiment with different forms of engaging stakeholders, project governance and methods. In this book, I have selected the two co-creation processes from Bremen for an in-depth analysis and the

${ }^{1}$ https://mobile-age.eu/. 
co-creation process from Zaragoza as a comparative case. The reason is that both, Bremen and Zaragoza, had a focus on information services concerning the neighbourhood in urban settings by relating to policy objectives such as age-friendly cities and communities. The projects ran over a similar length of time while following different governance structures, engagement strategies and co-creation methods. There is hence enough commonality and difference to provide a meaningful comparison between the three projects in relation to the sharing of control and expertise as well as the enabling of change.

\section{A Framework for Co-creation}

A co-creation project can be roughly split into four phases (1) a planning phase in which a problem focus of the project is determined; (2) a phase in which the problem area is explored and co-creators decide on a joint problem definition and articulate a value proposition; (3) a phase that develops and evaluates possible solutions; and (4) the post-project phase in which the service runs (Fig. 1). The chapters that report on our co-creation projects in Bremen and Zaragoza are concerned with the second phase (find the right problem) and third phase (find the right solution). Because of the logic of funded research projects, much of the planning had to be done prior to the project start for the funding proposal (see also Bischof \& Jarke, forthcoming). In a first attempt to receive project funding, we left the planning deliberately open in order to increase the scope for action in the co-creation projects. This was however rejected by the funding agency because proposals needed to be very specific on how and why they would spend taxpayer money. In the project proposal that received funding, we provided a frame for the problem focus, the problem definition itself was co-created with older adults, social care service providers and local governments during the co-creation projects. The running of the service also lies outside of the scope of a funded research project. However, we allowed ample time to ensure the sustainability of the services developed. For now,

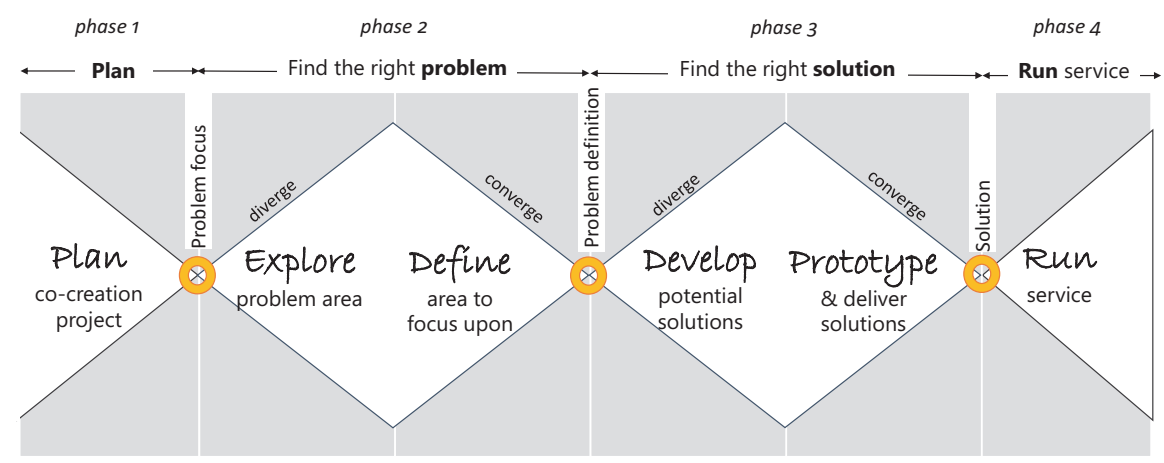

Fig. 1 Four phases of a co-creation project 


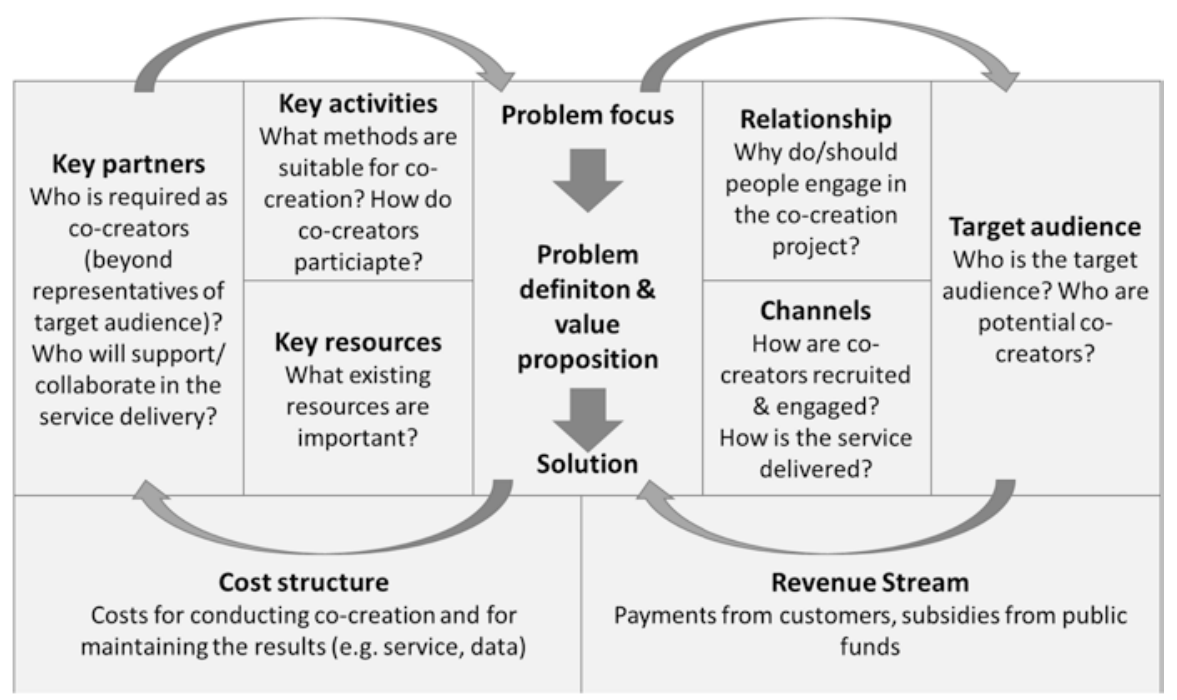

Fig. 2 Adapted business model canvas to describe co-creation process and output

I outline the results of the first phase (the problem focus) as this had implications for the choice and adaptation of methods in the subsequent phases.

When considering and planning a co-creation project (phase 1) there are a number of aspects to consider which frame and determine the project. Similar to a business model canvas one can depict the key dimensions of a co-creation project in a canvas (see Fig. 2). Initially, there needs to be a general idea about the problem focus and target audience. As the co-creation project proceeds the problem focus will become more refined (phase 2-find the right problem) and a solution is being developed (phase 3-find the right solution). This process is at the heart of cocreation and shaped by those engaged from the target audience and by key partners. The role that co-creators assume in a co-creation project depends on the relationship they have to the problem area and their willingness and ability to become engaged. Another relevant aspect are the communication and engagement channels (e.g. how citizens are approached and recruited to the co-creation project; how the service will be delivered). In addition, it is important to consider the existing resources (e.g. of a neighbourhood) that may support a service and what kind of activities are suitable and required in order to engage with the target audience and key partners in a meaningful and targeted way. For the sustainability and maintenance of the proposed solution (phase 4), the revenue streams and cost structure need to be evaluated.

These aspects are described in more detailed in the following. I will start with the problem focus of Mobile Age and detail the specific foci of Bremen and Zaragoza. Subsequently the target audiences and key partners are described. I provide a summary of key resources and subsequently refine the problem definitions \& value propositions. This includes a summary of the solution: output, outcome and sustainability of the co-created services. Finally, key activities are reviewed considering how 
methods from e.g. co-production, co-design and civic open data use may be adopted to suit the needs of our co-creation projects. The chapter concludes with a summary of the Mobile Age co-creation methodology.

\section{Problem Focus}

One of the basic assumptions of Mobile Age was that if digital services are more relevant to older adults and more user-friendly, they may raise interest in and use of digital public services (even for those with little or no digital skills). The corresponding term in the digital agendas of the European Commission and EU Member States is "e-inclusion". The main reason for political action in the field of e-inclusion is the risk of excluding those citizens that are not digitally literate and do not use digital media. In other words e-exclusion increases social exclusion. Thus, social inclusion and e-inclusion were two mutually depending policy aims of the Mobile Age project and the co-creation processes. The co-creation processes described in this book focused in particular on "social participation" (Bremen) and "a safe and accessible city for older citizens" (Zaragoza).

\section{Problem Focus: Social Participation (Bremen)}

Social participation is a societal (or political) goal that aims to enable any personno matter what age - to participate in the social, political, economic and cultural life (Naegele, Olbermann, \& Kuhlmann, 2016). It hence links to the promotion of citizens' empowerment and participation (as individuals, as groups or communities). Factors that may hinder the social participation of people are a "combination of linked problems such as unemployment, poor skills, low incomes, poor housing, high crime, poor health and family breakdown" (Lyons \& Huegler, 2013).

While there are several policy-related measures on the macro-level aiming at social participation (e.g. social policies, labour market reforms), the focus of Mobile Age was on the meso-level (neighbourhoods \& districts) and micro-level (individual \& families). On an individual level, social participation may be understood as participation in (Naegele et al., 2016, p. 45):

- economic (participation in work life, sufficient financial funds and right to make decisions);

- political (participation, civic engagement, possibility for decision making);

- cultural (access to cultural life and related education);

- social (informal and personal integration in primary networks such as family, friends and social activities in society;

- socio spatial (relationship and bond with respective living environment: happiness, identity, bonding). 
The British ELSA report proposes the term "social detachment" to measure the disadvantage on six indicators of social participation: (1) contacts with other people, (2) social support, (3) civic/political involvement, (4) participation in culture, (5) participation in recreational activities/hobbies and (6) participation in leisure (Banks, Breeze, Lessof, \& Nazroo, 2008; Tomaszewski \& Barnes, n.d.). In this perspective, improving social participation is meant to be achieved by strengthening social capital and circumventing social detachment through appropriate neighbourhood development. The neighbourhood not only affects outcomes such as education, employment and health, but also the opportunities for building social capital (Atkinson \& Kintrea, 2000). Enabling older adults to remain in their communities and neighbourhoods allows them (1) to connect and interact with other locals and to be part of a "safety net of people who look out for you and would come if something was wrong", and (2) know "where specific resources (e.g. health services and shops) are and how they work" (Wiles, Leibing, Guberman, Reeve, \& Allen, 2012).

The study conducted by Wiles et al. characterises "ageing in place" by the positive perceptions of older adults as a sense of attachment and social connection, a sense of security and familiarity and a sense of identity, linked to independence and autonomy. In the WHO framework, social participation is an issue of neighbourhood development in terms of public infrastructure, the availability and quality of local institutions and services in each of the action areas. To assist older adults to remain in their communities and neighbourhoods with some level of independence, rather than in residential care homes, requires to consider not only their immediate housing options but also "transportation, recreational opportunities, and amenities that facilitate physical activity, social interaction, cultural engagement, and ongoing education" (Wiles et al., 2012).

Hence, neighbourhoods play a central role for social participation/inclusion as social exclusion is often concentrated in certain neighbourhoods of a city or region (Ellen \& Turner, 1997; Pickett \& Pearl, 2001). Some European governments (e.g. Germany, UK) fund so-called neighbourhood managers in deprived communities who among other things collect:

- Evidence of residents' identified needs and priorities, and

- Evidence of the quality, level and performance of local public services and any gaps in provision or issues with performance.

They are also called "pathfinders", as one of their functions is to give evidencebased recommendations to local government which services in their neighbourhoods are missing or are of poor quality in order to improve social cohesion and combat poverty. A British evaluation report found that from the experience of neighbourhood management pathfinders it has become clear that

$[\ldots]$ baseline information at neighbourhood level is not always available, or not very accessible $[\ldots]$ improving information about levels of service and service expenditure at neighbourhood level continues to be a challenge (The National Evaluation of the Pathfinder Programme, 2006, p. 14). 
Social participation can hence be understood as an interplay between the resources of a neighbourhood and the resources of older people living there. People with low social and cultural capital, little financial resources and poor health will use the local resources to a lesser degree and participate less in (public) social life. If there is a lack of services and facilities in a neighbourhood even a high degree of personal resources does not lead to high a degree of social participation. Appropriate information about the available resources in a neighbourhood can have a positive effect on social inclusion, if it meets the media habits and abilities of the target audience.

\section{Problem Focus: Safe and Accessible City for Older Citizens (Zaragoza)}

In Zaragoza, the problem focus was specifically on a safe and accessible city for older citizens. The rationale for Zaragoza's problem focus was linked to the idea of "ageing in place" and the importance of neighbourhood for older residents as described for Bremen. It was also linked to Zaragoza's strategic policy objectives of becoming a WHO age-friendly city.

On March 27, 2009, the City Council unanimously approved the integration of Zaragoza into the WHO Global Age-Friendly Cities and Communities Network (GNAFCC). In March 2011, the accession to the network was formalised, with Zaragoza being the second Spanish city to be included. In a first phase of work, a "participatory diagnosis process" about high-impact areas was carried out using a methodology established in accordance with the Vancouver Protocol (InvestigationParticipation-Action). This diagnosis (or baseline report) included quantitative and qualitative research on the city, taking into account the eight different WHO areas. In the process, the city government collected information on every aspect of the city affecting older adults, and subsequently conducted focus groups with older citizens to seek their opinion on this information. The mechanisms and areas of participation of senior citizens in the process were defined in order to develop a diagnosis that would allow to measure the age-friendliness of the city with older people involving the participants in the analysis process and improvement proposals regarding programs, services and characteristics of the city for its older residents and to generate a series of proposals that would allow a plan of action adapted to the needs and demands of older citizens.

The development of the first phase of action generated a diagnostic document of the city, which not only evaluated its age-friendliness, but also analysed in each of the areas defined in the Vancouver Protocol the strengths and weaknesses in the opinion of its older residents, in addition to proposing improvement actions.

This information allowed the development of 25 proposals, with different levels of detail. These proposals informed an Action Plan. One of the projects in this Action Plan concerned "Walk and discover a safe and accessible city", relating to the articulated needs of older adults to increase security and accessibility in the city. 
Participants in focus groups proposed the co-creation of "age-friendly routes" which could be digitised and accessed through the City Council's web page. An age-friendly route was defined as follows:

- The route is frequently used: Co-creators need to identify a route that older residents use routinely and often in their daily life in the neighbourhood. The Senior Citizens Centre of the district was taken as a point of reference.

- The route is safe and accessible: Co-creators need to identify and assess aspects that make routes safe/unsafe and accessible/inaccessible.

\section{Target Audiences and Key Partners}

At the start of the Mobile Age project, the target audience was defined as older adults ( $>60)$. In chapter "Ageing Societies and Technological Innovation", I argued that even though older adults are not a homogeneous group and differ for example with regard to their personal resources (e.g. social capital, financial resources), aspirations and abilities, it does make sense to group them as a "target audience" of public services-based on life events-and of digital public services-based on their birth-cohort shared experiences with (media) technologies.

A digital application on its own cannot solve social problems. As discussed in chapter "Ageing Societies and Technological Innovation", the striving to "fix" social problems through technology, produces its own manifestations of these "problems" (e.g. old age as problem that needs to be fixed). Rather, a digital information service can only complement and inform about existing (neighbourhood) resources and/or support local service providers in their service provision. Hence, the target audience of a digital information service, mainly includes those older adults who are also targeted by the resources it provides information about. People in their Fourth Age (Laslett, 1987, 1991) can benefit from neighbourhood-related digital information services when intermediaries (such as family and service providers) are considered as well. For example in the case of Bremen, neighbourhood managers, as well as community managers and social care service providers can use digital information services in the communication with older adults. Apart from older adults as co-creators broadly representing the target audience, other key partners had to be involved in order to develop a service, which is comprehensive, sustainable and embedded in the neighbourhood.

The roles as depicted in Fig. 3 are partly overlapping. Intermediaries serve as information brokers and provide information about services, events and resources in digital or printed form to different groups of older adults. They may be professional neighbourhood managers, social workers in the field of elderly care or volunteers in community building, editors of community newsletters or city web portals, but also family members and acquaintances. In addition to older adults, intermediaries are the second target audience and user group of the services to be developed (as it should support their daily work). Considering the digital divide, they play an 


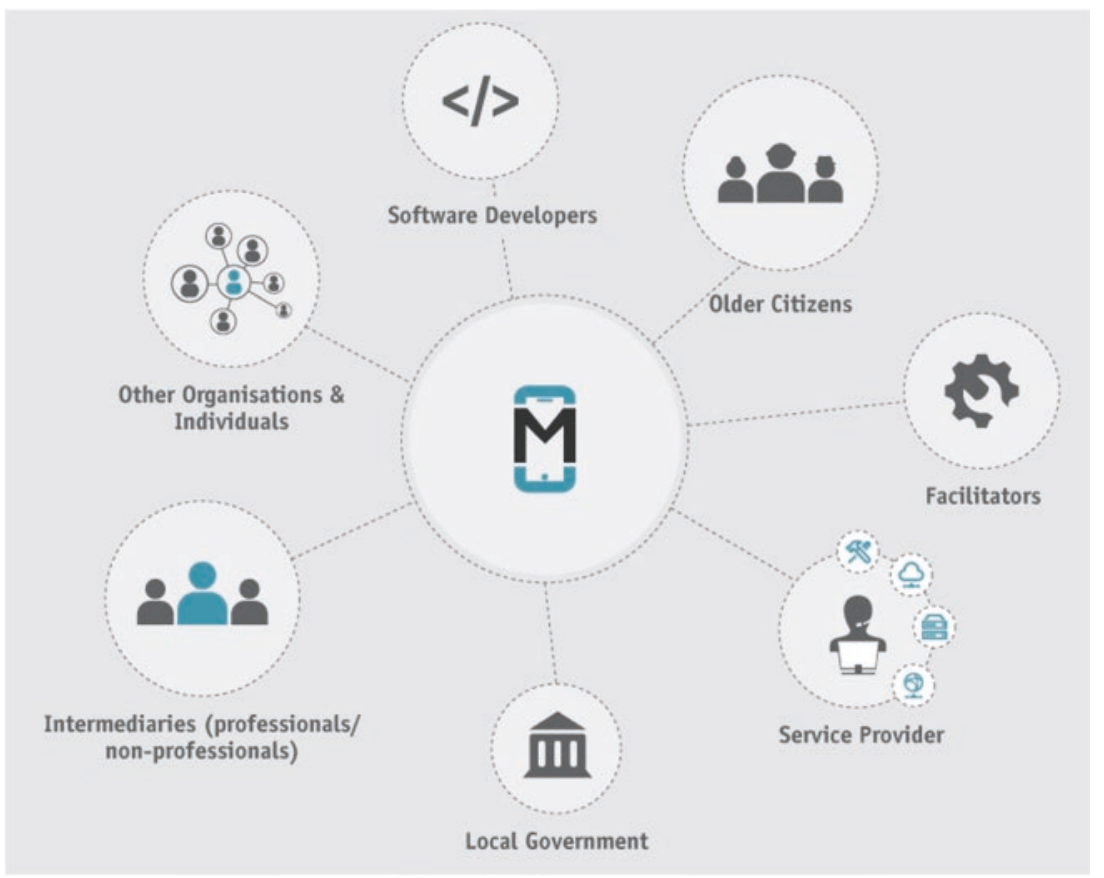

Fig. 3 Stakeholders in co-creation of open data-based public services

important role in making the content of a co-created digital service available to older citizens. It is hence important, that they provide input for specific tasks in the co-creation process.

Social care service providers are offering services to older adults in the neighbourhood. They include government units, utilities such as transport providers, social welfare organisations, religious congregations, NGOs, and commercial business (cafés, pharmacies etc.). They are the subject of the information service to be co-created. They may provide information about themselves and the details of their services and they have to agree to the publication of these data. In many cases, local/ regional government and public administrations will be initiating and managing the co-creation activities to provide financial resources, become the owner of the new service and maintain it.

A co-creation process needs facilitators as convenors and moderators. Facilitators in Mobile Age were either researchers (in Bremen) or experienced individuals in the work with older adults and/or groups (in Zaragoza). They supported the co-creation activities through, for example, running workshops, focus groups, and interviews. A digital service needs to be developed by software developers. Developing a usercentred application may be undertaken by IT-departments of the local government (Zaragoza), research institutes (Bremen), commercial companies or civil society organisations such as the Open Knowledge Foundation. 
Finally there are other organisations \& individuals that have to be engaged in order to provide missing information, financial resources or support the use and outreach of the service, including for example senior citizens' organisations, senior citizens' clubs (e.g. computer clubs) but also media and journalists that may report about their co-creation activities and the service and politicians engaged on social policy and elderly care.

\section{Key Resources: Information About the Neighbourhood}

Overall, the problem focus and domains of interest in the Mobile Age project related to different domains for age-friendly cities and communities as proposed by the World Health Organisation (WHO). Between the pilot sites, we had an overlap of topics as Bremen and Zaragoza shared an interest in map-based services and the importance of local infrastructure for supporting ageing-in-place. These services related to digital information and communication services (and not transaction or integration) about existing neighbourhood resources.

Figure 4 depicts the interrelation of age-friendly neighbourhood resources and the personal resources of its older residents. There exists a plethora of information about the resources of a neighbourhood, however in order to participate in social life and use those resources, they need to be accessible to older adults. A digital service

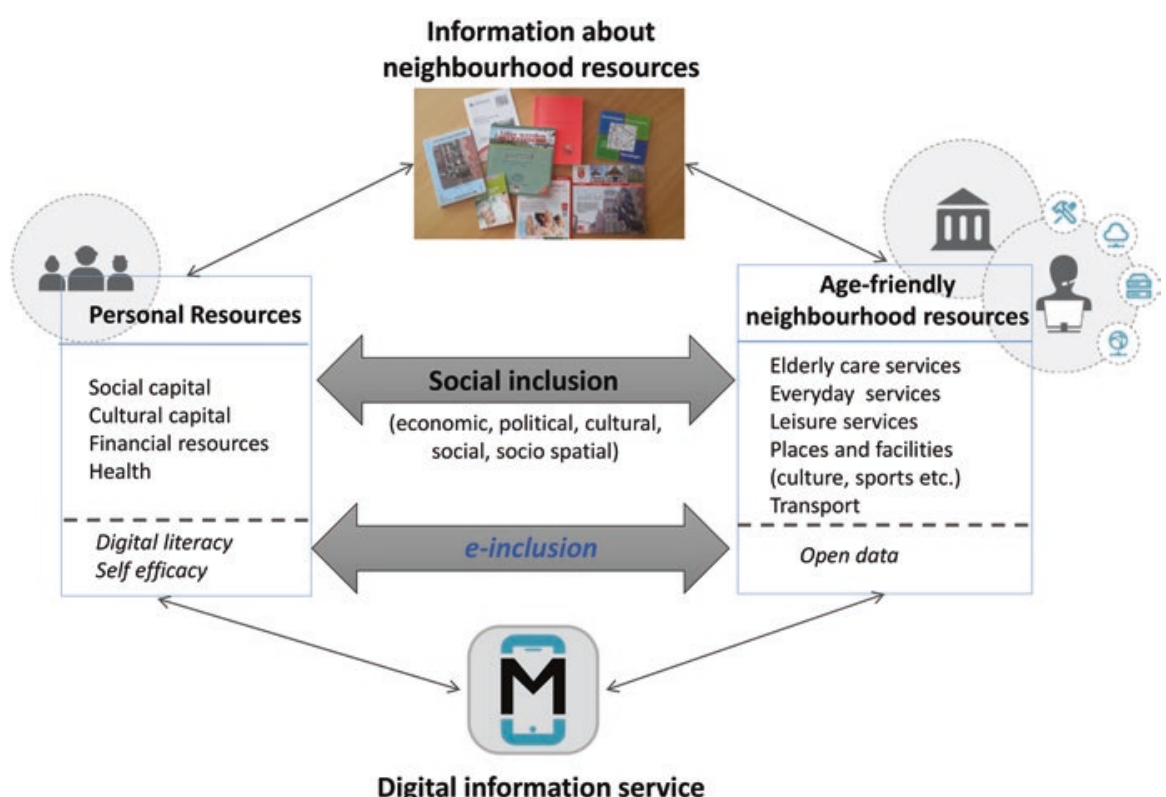

Fig. 4 Co-creation of a digital public information service as conducted in Bremen 
may complement the information about these resources (but cannot substitute for them).

For engaging senior citizens to co-create a digital service that meets their needs and that offers gratifications to a larger group of older adults, information about the resources in their immediate neighbourhood has proven to be a good starting point. The co-creation process as conducted in Zaragoza also allowed for a communication service in that the citizens could propose changes to the built environment (addition grey arrows) (Fig. 5).

\section{Problem Definition and Value Proposition}

The value proposition summarises in a few sentences in which respect the planned service will solve a particular problem and in which respect it is better than existing services. In most cases, a service may provide benefits not only to older adults, but also relieve family members or caretakers and/or save the costs of service provision for local government or social welfare organisations. Therefore, it is important that for each target audience a value proposition is defined. The problem definitions and value propositions for each of the three co-creation projects are described in detail in the respective chapters.

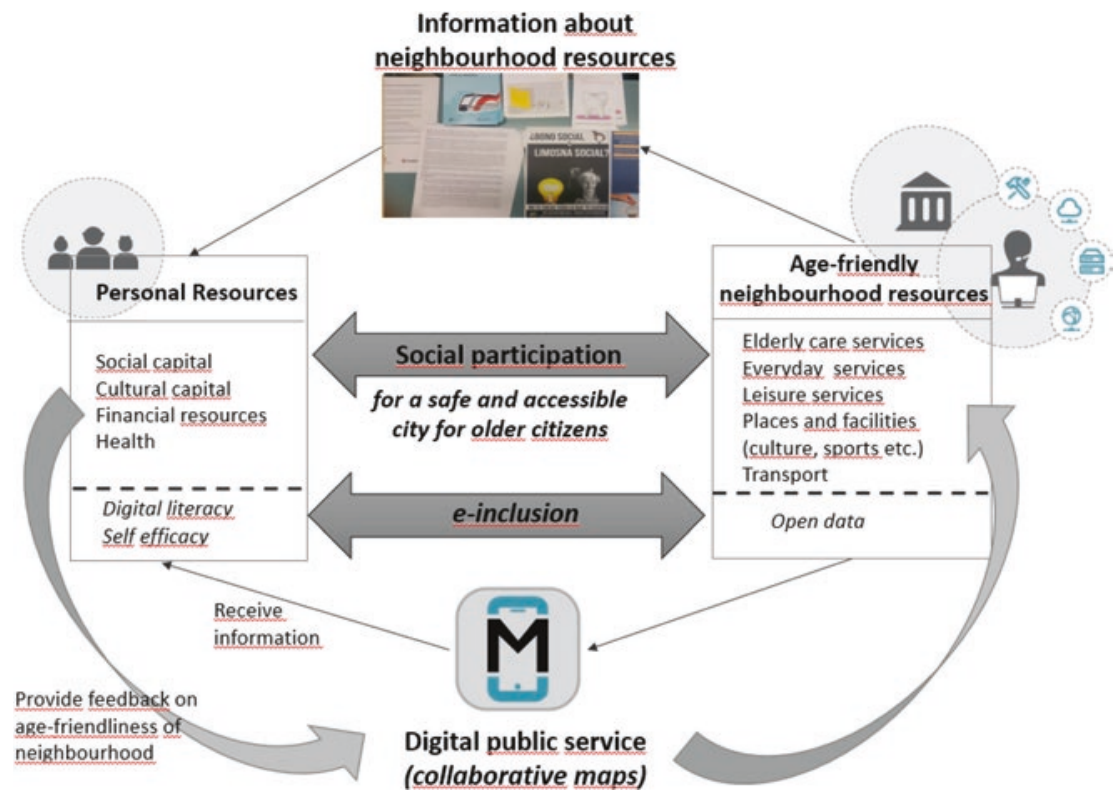

Fig. 5 Co-creation of digital public service allowing information and communication as conducted in Zaragoza 


\section{Solution: Output, Outcome and Sustainability}

In Mobile Age, we defined the co-created services as "solution" to the co-created problem definitions. The "output" of the projects were a demonstrator app and the co-created data, the usage of the service is its "outcome" in quantitative and qualitative terms (e.g. number of users and immediate benefits). Depending on who provides the service, quite different outcomes are to be expected. For example, in Zaragoza the map-based service was part of the local government's online service portal from the beginning and was continued after the project without any interruption or modification. In contrast, in Bremen, the map-based district guides were developed by the Mobile Age technical partner FTB on their own server and wereafter several months of pilot operation-migrated to the city's information portal featuring the same data, similar functionality and look \& feel. A more detailed account on the output and outcome of the each of the co-creation projects is provided in the subsequent chapters.

\section{Expected Impact}

The usage of the new digital public services is an important aim in itself but in the context of public services at the same time a means to achieve more general social policy objectives such as social participation or age-friendly environments. These kinds of impacts are difficult to assess but an important argument for local governments to invest in the co-creation such digital services. However, it is possible to collect assessments by different key partners involved in the respective fields of elderly care and social work as well as local government. This assessment is provided for each of the three co-creation projects in the subsequent chapters.

\section{Key Activities: Adopting Methods for Co-creation with Older Adults}

In Mobile Age, we identified a number of streams of activity that need to be considered for the co-creation of digital public services. These streams of activity are not sequential but run in parallel and inform each other.

- The first stream of activity concerns the governing and managing of a co-creation process. This includes the exploring and scoping of the project, the planning of resources as well as considerations about ethics.

- The second stream of activity covers the continuous recruitment and engagement of stakeholders throughout the co-creation process.

- The third stream concerns the co-creation of a service concept. This includes the development of ideas about the service to be co-created based on the needs and requirements of older citizens and intermediaries, the definition of a (rough) service concept and the subsequent refinement of this concept. This is based on approaches to co-producing public services. 
- The fourth stream is concerned with (open) data. It includes the identification of existing and missing data, the collection, validation and quality checking of data, the creation and integration of open data as well as the editing of data and information. This is grounded in work on civic open data use.

- The fifth stream is concerned with the co-creation of software. This includes the identification of desired functionalities, prototyping and user testing and is based in approaches to co-design.

- The sixth stream of activity concerns evaluating the co-creation process and its results. This is a continuous activity throughout the whole process and includes formative as well summative evaluation.

- In addition, a co-creation process needs to include activities pertaining to exploitation and dissemination. Finally, the service provision needs to be considered.

These streams of activity need to facilitate the sharing of knowledge and control amongst participants and across participants and facilitators as well as enable opportunities for individual and social change. In the co-design literature, there exists a manifold of research papers and studies presenting tools and methods to involve users through e.g. cultural probes (e.g. Boehner, Vertesi, Sengers, \& Dourish, 2007; Jarke \& Maß, 2018); personas and scenario-based design (e.g. Alexander \& Maiden, 2004; Carroll, 2000; Neate, Bourazeri, Roper, Stumpf, \& Wilson, 2019) or walks (e.g. Hunter, 2018; Kanstrup, Bertelsen, \& Østergaard Madsen, 2014). However, there is only limited experience in the adaptation of these methods for digital public service design, which comes with its specific requirements in terms of scalability, accountability and sustainability.

One of the most common ways of eliciting users' expertise in co-production as well as co-design projects are workshops in which teams of researchers, service providers, future users and other stakeholders come together to identify challenges and develop new ideas. Depending on the design context and the quality of user participation, the interpretative weight of the design team differs. For example, Bødker, Grønbæk, and Kyng (2012) doubt whether personas are helpful in designing public services because those defining the personas cannot really comprehend and represent the heterogeneity of the target population and future users. This is in line with reports that in the case of user participation in government services, government officials doubt the relation between user participation and later acceptance because nobody can tell to which extent the people who are interested to participate represent the target user group of a service (Gidlund, 2012). Overall, there are three challenges to user participation in digital public service design, that need to be considered: (1) the target user (segments) need to be identified clearly; (2) the modes of participation need to be in line with democratic goals and (3) if citizens lack sufficient ability and skills the successful outcome may be endangered (Karlsson, Holgersson, Söderström, \& Hedström, 2012).

Each of the phases in a co-creation process has its own conditions; they each require different types of methods. Figure 6 provides an overview about the four distinct stages of co-creating a digital public service (as described in Fig. 1): its planning, its design (defining the problem and developing a solution) and its 


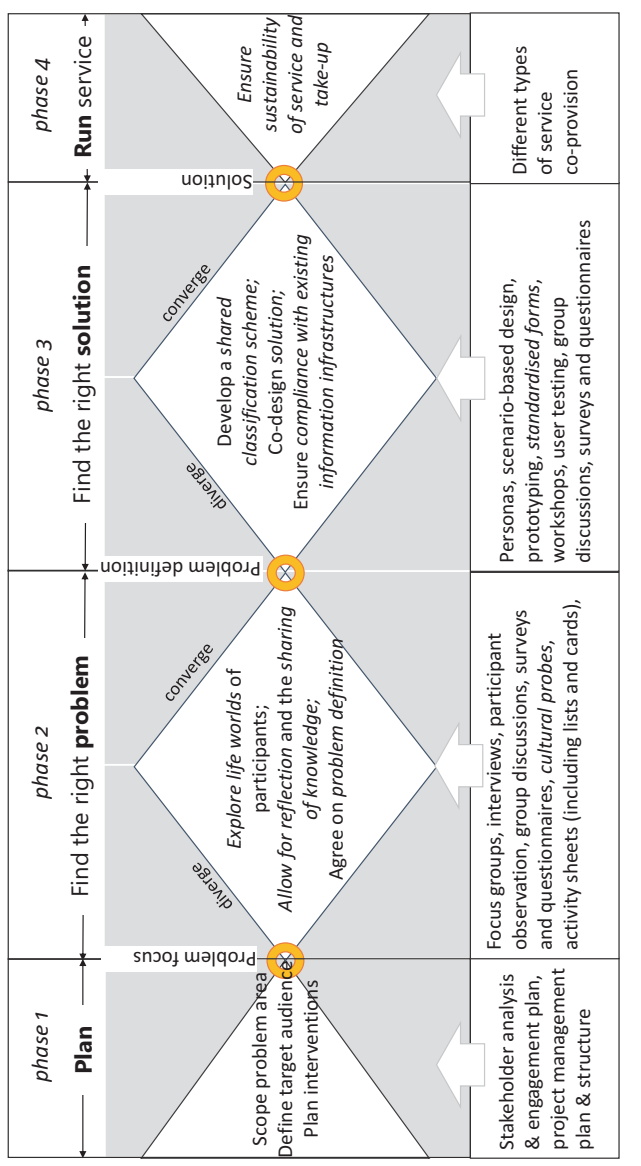

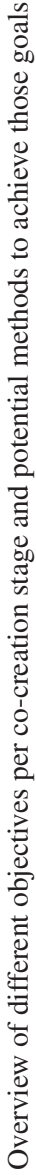


provision. The figure lists some indicative methods for each of the phases and related objectives. A detailed description of each of the methods can be found in the interactive guidebook we developed after the finalisation of the Mobile Age project. ${ }^{2}$

The abstract concepts of use and user as promoted in many participatory projects, encourage particular practices while discouraging others (Gidlund, 2012, p. 18). It is hence important to consider how different methods produce different subject-positions of citizens as co-creators. Interviews, focus groups, prototyping or user testing are well-known methods in design projects and power relations have been well-covered (e.g. Carroll, 2000; Carroll \& Rosson, 2007; Neate et al., 2019; Oates, 2006; Sanders \& Stappers, 2008; Simonsen \& Robertson, 2013). In the following, attention is paid to methods that are less known for their use in co-creation settings and it is explored how they can be adapted to contribute to the objectives described above in Fig. 6:

- Probes as method for

- exploring life worlds of participants;

- allowing for reflection and the sharing of knowledge;

- developing a shared problem definition.

- Standardised forms as method for

- developing shared classification schemes;

- co-designing solutions.

- Data walks as method for

- exploring life worlds of participants;

- allowing for reflection and the sharing of knowledge;

- developing a shared problem definition;

- developing shared classification schemes;

- co-designing solutions.

The focus is on phases 2 (find the right problem) and 3 (find the right solution). Probes, standardised forms and data walks are introduced below and their application in Mobile Age described in the respective chapters reporting from our co-creation projects in Bremen Osterholz (probes and standardised forms), Bremen Hemelingen and Zaragoza (walks and standardised forms). In chapter "Learning from Co-Creation Practice", the methods are analysed and discussed across the three co-creation projects, considering their suitability for facilitating the sharing of power, the sharing of expertise and the enabling of individual and social change in co-creation with older adults.

\footnotetext{
${ }^{2}$ https://co-creation.mobile-age.eu/.
} 


\section{Probes $^{3}$}

Probes were originally conceived by a group of researchers/designers within an EU-funded project to engage older adults in user-centred design (Gaver, Dunne, \& Pacenti, 1999): The cultural probes_a pack of maps, postcards, a camera, a photo album and media diary_-were designed to provoke inspirational responses from elderly people in diverse communities" (p. 22). Gaver et al. conceived of probes as something like astronomic or surgical probes, which are left behind when researchers leave and over time return fragmentary data. The probes were part of an experimental design, in which a group of researchers wanted to explore new ways for designing technology for unfamiliar user groups.

Understanding the local cultures was necessary so that our designs wouldn't seem irrelevant or arrogant, but we didn't want the groups to constrain our designs unduly by focusing on needs or desires they already understood (Gaver et al., 1999, p. 22).

In contrast to scientific probes, cultural probes were meant to be a source for inspiration, not information. The approach aims to be a resource for surprise and creativity.

In subsequent years, probes became widely adopted in user-centred and participatory design and were amended to include concepts such as "design probes" (Mattelmäki, 2006), "technology probes" (Hutchinson et al., 2003), "mobile probes" (Hulkko, Mattelmäki, Virtanen, \& Keinonen, 2004) or "digital probes” (Koch \& Maaß, 2018). For Boehner, Gaver, and Boucher (2012); Boehner et al. (2007) probes are different from other social research methods as they embrace uncertainty and ambiguity and therefore invite interpretations by designers and participants:

They [probes] aim to open up possibilities, rather than converging towards singular truths, and can be understood as part of a conversation among designers and the people and places for which they design (Boehner et al., 2012, p. 185).

One way in which probes came to be appropriated was as a tool for data collection. Most studies, as Boehner et al. (2007) point out in their review, adopt probes as part of their initial investigation for understanding a particular use context. Often they are coupled with interviews and at times supplement ethnographic approaches. Some studies integrate probes in participatory design exercises; for example, similar to our approach in Mobile Age, Maaß and Buchmüller (2018) discuss the probe returns with participants. Hence across different use contexts, probes are either used to understand current use situations or for ideation and prototyping (Jarke \& Maaß, 2018).

Some studies take the participatory aspects of probes further and insist that participants should also be involved in the translation of the probes into design ideas (Boehner et al., 2007, p. 1079). Others see probes as a possibility to allow participants to reflect on their own practices and to express these reflections (Boehner et al., 2007; Vetere, Davis, Gibbs, Francis, \& Howard, 2006, p. 1477). Participants decide and control what information they record and share, and in so doing secure their privacy.

\footnotetext{
${ }^{3}$ This section is part of an article published by Ulrike Gerhard and myself (Jarke \& Gerhard, 2018).
} 
Importantly are probes not an alternative formal or objective method for simply "getting data" but rather "frame an alternative account of knowledge production in HCI design" (Boehner et al., 2007, p. 1078). In their review of how HCI researchers have appropriated probes, Boehner et al. (2007) suggest that there has been a shift in the definition and interpretation of probes from response to representation: "from seeing interpretation as a researcher responding to what was expressed by the researched to seeing interpretation as a researcher ascertaining facts about the research" (p. 1082). The idea of interpretation as response understands the process as dialogical in the sense that researchers articulate their research questions and instruments, which are interpreted by the participants. The participants in turn respond by expressing their interpretations; researchers respond by expressing their interpretations through potential design ideas. There is never an attempt to "fix the true meaning of any particular response". In contrast, the idea of interpretation as representation aims to "fix the true meaning of what users said, who they are, what they do, and what they need" (p. 1083). Boehner et al. (2007) criticise that "a major focus of probes' uptake in HCI has been to use probe returns to develop objective, factual descriptions of user needs" (ibid).

In the context of co-creation, I want to propose a different understanding of the role of interpretation when using probes. Probes as boundary objects that enable/ facilitate the articulation of users' tacit knowing and the shared interpretation of their accounts. Others have pointed to the ability of probes to act as "boundary objects" (e.g. Bjögvinsson, Ehn, \& Hillgren, 2012; Ehn, 2008). What is of particular interest for this book are the ways in which probes allow for collaboration and sharing of knowledge across social worlds and facilitate the ways in which expertise is being negotiated and made accessible across communities of practice.

\section{Standardised Forms}

While probes may serve as boundary objects for sharing expertise and knowledge, standardised forms are more structured boundary objects that facilitate other dimensions in co-creation processes. Standardised forms (along with methods and procedures which enforce normative practices across knowledge boundaries) provide a shared format for solving problems. They are one way to attend to the challenges posed by co-creating shared classifications and developing solutions. These objects can circulate easily and provide a standardised way of collecting information (Star \& Griesemer, 1989). What is hence required are interventions that allow for the development and use of standardised forms in order to complement open methods such as probes. Standardised forms are for examples tables or templates in which data (and information) are collected. Within co-creation the structure of these tables or templates - the types of objects and their attributes — should be co-developed by citizens and reflect their life worlds.

\section{Data Walks ${ }^{4}$}

Walking is a human activity, engrained in urban and rural culture. It is also becoming a prominent method in projects related to co-design and critical data studies

${ }^{4}$ This section is part of an article published by myself in (Jarke, 2019). 
(Wieringa \& van Es, 2018) as well as participatory design (Kanstrup et al., 2014). What makes walks an interesting and appealing tool for engaging older citizens (critically) with data is their embeddedness in everyday urban life. Data walks have been proposed and conducted in a number of projects aiming to engage with data and putting an 'emphasis on the everyday experience of data' (Wieringa \& van Es, 2018) as well as the relationality of design (Kanstrup et al., 2014).

Wieringa and van Es (2018) have mapped a number of different formats each comprising of different set-ups and goals. For example Greenfield and Kim (2011) set out to raise awareness/literacy on "networked urbanism" among citizens. Van Zoonen, Hirzalla, Engelbert, Zuijderwijk, and Schokker (2017) took city employees on walks through their own smart city. While Greenfield and Kim only delimited an area on a map, Van Zoonen et al. defined the routes beforehand. The focus of their walks was "identifying big data in the city and connecting it to political and ethical issues" (Wieringa \& van Es, 2018). In so doing, Van Zoonen et al. not only raised awareness on data issues amongst civil servants, they also learned about the knowledge and beliefs of their participants with respect to the datafication of their city. Building on Greenfield and Kim, Powell (2018) experimented with different forms of data walks: initially to teach students about big data related to urban issues, later to create "bottom-up knowledge". In her walks, participants assumed different roles from note-taker to photographers. Yet another format of data walks was conducted by Hunter (2018), who did not only want to raise awareness amongst participants but also collect environmental data on specific areas and built multi-layered "dataspaces". Table 1 provides an overview on these different types of walks.

In sum, one question that civic open data approaches such as data walks allow to ask is what kind of (digital) information are relevant and useful to (a variety of) older adults and what kinds of data, data visualisations and data processing (e.g. filtering, searching) are required in order to provide information about neighbourhoods. These are questions that relate to the ways in which information about neighbourhoods are being classified and presented in e.g. pocket guides, catalogues or leaflets.

\section{Summary}

Probes, standardised forms and walks are methods that were developed in specific participation approaches: cultural probes are derived from design research, standardised forms from knowledge management research and different types of walks are deployed in various forms of civic open data use. The next three chapters describe in detail how we conducted our three co-creation projects and how these methods were amended to suit our co-creation needs. The chapters feature all the other methods we adopted such as interviews, focus groups, questionnaires, card games, co-design workshops, paper and digital prototyping, and user tests. In chapter "Learning from Co-Creation Practice", these methods are analysed and discussed with respect to enabling us to share control and knowledge with and amongst different groups of co-creators, and ultimately facilitating individual and social change. 
Table 1 Inventory of different data walks (excerpt from Wieringa \& van Es, 2018)

\begin{tabular}{|c|c|c|c|c|}
\hline & $\begin{array}{l}\text { Greenfield and } \\
\text { Kim (2011) }\end{array}$ & Powell (2018) & $\begin{array}{l}\text { Van Zoonen et al. } \\
\text { (2017) }\end{array}$ & Hunter (2018) \\
\hline Goals & $\begin{array}{l}\text { Raising awareness/ } \\
\text { literacy on } \\
\text { 'networked } \\
\text { urbanism' }\end{array}$ & $\begin{array}{l}\text { Originally } \\
\text { teaching tool } \\
\text { against } \\
\text { celebratory } \\
\text { rhetoric. Now } \\
\text { raising awareness } \\
\text { and 'creating } \\
\text { bottom-up } \\
\text { knowledge' }\end{array}$ & $\begin{array}{l}\text { Gaining insight into } \\
\text { civil servants' ideas } \\
\text { and beliefs about } \\
\text { datafication and } \\
\text { 'strengthening their } \\
\text { critical interrogative } \\
\text { attitude' }\end{array}$ & $\begin{array}{l}\text { Examining tools } \\
\text { and technology } \\
\text { for data } \\
\text { collection, and } \\
\text { experimenting } \\
\text { with data } \\
\text { visualization }\end{array}$ \\
\hline $\begin{array}{l}\text { Number of } \\
\text { participants } \\
\text { per walk }\end{array}$ & 15 & $\begin{array}{l}5 \text { per team (max. } \\
15)\end{array}$ & $4-6$ & - \\
\hline \multirow{5}{*}{$\begin{array}{l}\text { Role of } \\
\text { participants }\end{array}$} & Photographer & Navigator & Participants & \multirow[t]{5}{*}{ Data creator } \\
\hline & Map-maker & Photographer & \multirow{4}{*}{$\begin{array}{l}\text { Participant } \\
\text { observers }\end{array}$} & \\
\hline & \multirow[t]{3}{*}{ Note-taker } & Map-maker & & \\
\hline & & Note-taker & & \\
\hline & & Collector & & \\
\hline $\begin{array}{l}\text { Duration of } \\
\text { walk }\end{array}$ & $90 \mathrm{~min}$ & $60 \mathrm{~min}$ & $60 \mathrm{~min}$ & Different lengths \\
\hline $\begin{array}{l}\text { Duration of } \\
\text { event }\end{array}$ & Half a day & Half a day & $60 \mathrm{~min}$ & $\begin{array}{l}\text { Different lengths, } \\
\text { from } 3 \\
\text { days-45 min }\end{array}$ \\
\hline $\begin{array}{l}\text { Event } \\
\text { makeup }\end{array}$ & Walk-discussion & $\begin{array}{l}\text { Briefing-walk } \\
\text { critical making }\end{array}$ & Walk-debriefing & $\begin{array}{l}\text { Preparation- } \\
\text { walk- } \\
\text { visualisation }\end{array}$ \\
\hline Outcome & $\begin{array}{l}\text { Documentation of } \\
\text { walkshop and } \\
\text { online follow-up } \\
\text { with other walks } \\
\text { (e.g. through } \\
\text { hashtag) }\end{array}$ & $\begin{array}{l}\text { Direct participants } \\
\text { attention to } \\
\text { matters of concern } \\
\text { such as } \\
\text { surveillance, } \\
\text { ethics, urban } \\
\text { design }\end{array}$ & $\begin{array}{l}\text { Discover visible } \\
\text { and invisible urban } \\
\text { data infrastructure }\end{array}$ & $\begin{array}{l}\text { Visualisations of } \\
\text { walks }\end{array}$ \\
\hline
\end{tabular}

Open Access This chapter is licensed under the terms of the Creative Commons Attribution 4.0 International License (http://creativecommons.org/licenses/by/4.0/), which permits use, sharing, adaptation, distribution and reproduction in any medium or format, as long as you give appropriate credit to the original author(s) and the source, provide a link to the Creative Commons license and indicate if changes were made.

The images or other third party material in this chapter are included in the chapter's Creative Commons license, unless indicated otherwise in a credit line to the material. If material is not included in the chapter's Creative Commons license and your intended use is not permitted by statutory regulation or exceeds the permitted use, you will need to obtain permission directly from the copyright holder.

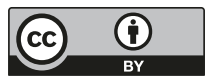

